Aus der Medizinischen Klinik der Universität in Tübingen. (Direktor: Prof. Dr. Romberg.)

\title{
Ueber vasomotorische Krampfzustände bei echter Angina pectoris.
}

\author{
Von Privatdozent Dr. Hans Curschmann, Assistenzarzt \\ der Klinik.
}

Während man den genuinen vasomotorischen Neurosen, den Akroparästhesien, der Erythromelalgie und ihren zahlreichen Uebergangsformen bis zum Raynaudschen Symptomenkomplex in neuerer Zeit das lebhafteste Interesse zugewandt hat, sind die angioneurotischen Krampfzustände der Peripherie als Begleiterscheinungen oder Aequivalente der Angina pectoris vera bisher viel $\mathrm{zu}$ wenig beachtet worden. Dabei ist die Kenntnis dieser Erscheinungen durchaus nicht neueren Datums.

Schon bei Romberg dem Aelteren finden wir sie angedeutet in der Schilderung, die er von dem äußeren Bild des Angina-pectorisAnfalls entwirft: "Die Temperatur der Hände und des Gesichts ist kühl, das Kolorit blaß; in den Fingerspitzen prickelnde Sensationen, wie beim Einschlafen der Glieder.“

Die erste eingehende und noch heute klassische Schilderung dieser Zustände gab Nothnagel in seiner Arbeit über die Angina pectoris vasomotoria. Ihm schließen sich eine Beobachtung Bambergers und in neuerer Zeit einige wenige Fälle von v. Schrötter und Huchard an. Auch Herz schildert in seiner zusammenfassenden Bearbeitung der Herzneurosen einen einschlägigen Fall. Pal, der in jüngster Zeit die vasomotorischen Krisen aufs eingehendste monographisch darstellte, bringt zu diesem Kapitel keine eigenen Beobachtungen.

Die neurologischen Bearbeiter der vasomotorischen Neurosen Frankl-Hochwart, Oppenheim und Cassierer haben auf den engen Zusammenhang der peripherischen Angiospasmen mit der Angina pectoris vera nicht speziell geachtet.

Es erscheint mir deshalb von mehr als kasuistischem Interesse, auf das genannte Syndrom an der Hand einiger eigenen Beobachtungen näher einzugehen. Vielleicht können sie auch als Anregung dienen, bei anscheinend genuinen vasomotorischen Neurosen mehr auf das Verhalten des Herzens und des Gefä̈rsystems zu achten, als dies bisher meist geschieht. Ich gebe die Schilderung dieser Fälle absichtlich mit der Ausführlichkeit, die $\mathrm{Krehl}^{1}$ ) an manchen der einschlägigen Darstellungen vermibte und mit Recht als notwendig gefordert hat.

Fall 1. Chronische Herzinsuffizienz. Angina pectoris vera mit angioneurotischen Krampfzuständen der Peripherie (obere Extremitäten, FüBe, Gesicht). Nephritis interstitialis chronica incipiens. Mors subita.

Frau Dorothea L., 58jährige Landwirtsfrau. Patientin klagt seit drei Jahren über zunehmende Herzbeschwerden, Herzklopfen, Engigkeit, Atemnot bei schwerer Arbeit und beim Steigen. Seitdem häufig leichte Anschwellungen der Füße und Abnahme der Urinmenge. Im letzten Vierteljahr wird Patientin besonders durch häufig, namentlich nachts wiederkehrende, heftige Schmerzanfälle beängstigt: unter starkem Druckgefühl auf der Brust, großer Angst, die sich bis zum Vernichtungsgefühl steigert, kommt es zu heftigen Schmerzen in der Herzgegend und vor allem in der Magengegend; die Schmerzen haben einen zusammenschnürenden Charakter. Während dieser Anfälle kommt es stets auch zu heftigen Schmerzen und Parästhesien in beiden Armen und Händen, seltener und geringer auch in den Füßen. Die Hände sollen dabei stets eiskalt und völlig gefühllos sein und eine bläulich-blasse Farbe haben. Aehnliche Veränderungen und Empfindungen hat sie auch im Gesicht beobachtet, besonders an Nase und Wangen. Nach einer Dauer von einer Viertelstunde bis 1-2 Stunden klingen alle Er- 
scheinungen (Herz- und Kauchschmerz, die „Krämpfe“ an den Extremitäten und im Gesicht) wieder ab. An den Händen (besonders der rechten) bleibt alsdann eine auffallende Röte und Hitze zurück, die in letzter Zeit fast permanent geworden sind. Patientin sieht dem Rezidivieren der Anfälle seitdem mit förmlich tödlicher Angst entgegen. Diese Anfälle treten nicht immer in dieser Heftigkeit auf. Bisweilen kommt es - besonders nach Kälteeinwirkung nur zur Asphyxie der Hände und des Gesichts unter mäBig beängstigenden Druckerscheinungen auf Brust und Magen. Keine besonderen Vorkrankheiten, kein Potatorium, kein Verdacht auf Lues. In der Familie keine ähnlichen Erkrankungen, keine Nervenleiden. Menopause seit sieben Jahren.

Status: Präsenile, fast kachektische Frau, ziemlich dyspnoisch; wegen der A temnot im Bett aufrecht sitzend. An Nase und Wangen umschriebene Cyanose, sonst gelblich-blasse Gesichts- und Hautfarbe. Die rechte Hand zeigt besonders auf der Dorsalseite eine großfleckige Röte, die linke Hand dieselbe in geringerem Maße. Gesicht und Extremităten kühl. Kein Fieber. Lungen: emphysematisch, links hinten unten zahlreiche bronchitische Geräusche. Das Herz ist beträchtlich von der Lunge überlagert. Orthopercutorisch reicht es nach rechts fingerbreit außerhalb des rechten Sternalrandes, nach links bis zur Mammillarlinie. Erregte Pulsation über dem ganzen rechten Herzen, Spitzenstoß verbreitet, hebend im V. Intercostalraum in der Mammillarlinie. Aktion sehr frequent (140 p. M.), irregulär, zahlreiche ventrikuläre Extrasystolen, die den Radialpuls zum Teil nicht erreichen. Töne: leise, meist rein, metallische Akzentuation des zweiten Aortentons. Der Radialpuls zeigt ein enges, rigides Rohr und trotz der Kleinheit eine vermehrte Spannung. Blutdruck (nach Riva-Rocci) $140 \mathrm{~mm} \mathrm{hg.}$

Abdomen meteoristisch aufgetrieben, starke Enteroptose, Leber überragt den R. b. d́reifingerbreit, induriert. Milz nicht palpabel.

Nervensystem: Hirnnerven ohne Veränderungen. Augenhintergrund ohne Veränderungen. Motilität normal. Sensibilität auch an den Extremitätenenden zurzeit normal. Haut- und Sehnenreflexe ohne Besonderheiten.

Urin: Menge reduziert, spezifisches Gewicht 1009, etwas trübe, spärlich Eiweiß, kein Zucker. Im Sediment zahlreiche Leukocyten, vereinzelte hyaline und granulierte Zylinder, Nierenepithelien. Kein Blut.

Ord.: Pil. Digitalis, viermal 0,05 , Veronal $0,3-0,5$.

Unter Digitalis besserten sich Frequenz und Qualität des Pulses in einigen Tagen unter langsamem Sinken des Blutdrucks auf $125 \mathrm{~mm}$ hg R. R., die Dyspnoe, die Oedeme und die allgemeine Schwäche wurden geringer. Die Diurese stieg bis auf $2000 \mathrm{ccm}$, die Beschaffenheit des Urins (Albumengehalt und spezifisches Ge. wicht) blieben unverändert.

26. Juli, morgens $81 / 2$ Uhr, hat Patientin plötzlich einen heftigen Anfall von präcordialem und abdominalem Schmerz mit dem ihr wohlbekannten intensiven Angstgefühl; zugleich nehmen beide Hände eine teils leichenblasse, teils cyanotische Färbung an (R $>\mathrm{L}$ ); die sonst intensiv roten Partien des Dorsums sind ausgesprochen cyanotisch. Beide Hände sind eiskalt; dabei hat Patientin heftige Schmerzen und Parästhesien in den asphyktischen Fingern, verbunden mit hochgradiger Hypästhesie gegen alle Qualitäten! an den Fingern. Im Gesicht haben die sonst umschrieben geröteten und leicht cyanotischen Partien an Wange und Nase eine blaßbläuliche Farbe angenommen; auch die FüBe sind bleicher und kälter als sonst und schmerzen. Der Puls stieg auf 112-116, fühlte sich härter an als sonst, war vielleicht etwas regulärer als sonst. Der Blutdruck stieg während des Anfalls a uf $165 \mathrm{~mm} \mathrm{hg} \mathrm{(sonst} 125 \mathrm{~mm}$ ). Die Atmung war eher etwas verlangsamt (28 p. M.) gegen früher. Die Temperatur stieg unter lebhaftem Frostgefühl auf 37,2 (gegen 36,3 bis 36,5 sonst).

Auf Morphin und warmen Wein in $1 / 2$ Stunde Nachlassen des Anfalls, die vorher blaß-cyanotischen Hände nahmen jetzt eine intensive fleckige Röte an $(R>L)$ und waren heiß. Der Puls hatte bald nach dem Anfall seine alte Frequenz wieder (88-92). Der Blutdruck war $1 \frac{1}{2}$ Stunden nach dem Anfall um $35 \mathrm{~mm}$, auf $130 \mathrm{~mm}$ gesunken.

26.-27. Juli. Nachts wieder sehr heftiger Anfall von Herzund Abdominalschmerz, Schmerz und totaler Asphyxie der Finger und des Gesichts, Vernichtungsgefühl; zwei Stunden Dauer. Dabei Frostgefühl und Temperaturanstieg, angeblich ohne besondere $\mathrm{Zu}$ nahme der Pulsfrequenz. Nach dem Anfall intensive Röte besonders der rechten Hand.

In den nächsten Nächten abortive Anfälle ganz ähnlicher Art. Die großfleckige Rötung der rechten Hand nimmt immer mehr zu. Das Allgemeinbefinden wird schlechter, die Stauungsbronchitis heftiger; es treten Temperaturen über $39^{\circ}$ auf, dazu ein eigentümliches diffuses, fleckiges Exanthem (Arzneiexanthem von Tct. Strophanti?)
Die Urinmenge sinkt etwas, Eiweibgehalt niedriger, spezifisches Gewicht 1011-1012, Sedimentbefund gleichgeblieben. Der Blutdruck ist lytisch auf 105 herabgesunken.

Nachts vom 4. bis 5. August sehr heftiger Angina-pectorisanfall mit Schmerzen in Händen und Füßen, verbunden mit totaler Asphyxie. Einige Stunden nach diesem Anfall - nach ruhigem Schlaf in erneutem Anfall - urplötzlich Exitus letalis.

Die Sektion (Dr. Heyde) ergab: Das Herz ist auffallend schlaff und weich, kautschukartig, es ist etwas größer als die Faust der Leiche. Die Vergrößerung betrifft insbesondere den rechten Ventrikel, der beträchtlich dilatiert und hypertrophisch ist; auch der linke Ventrikel ist etwas erweitert und hypertrophisch. Sämtliche Klappen dünn und zart. Die rechte Coronararterie frei von Veränderungen, dagegen zeigt die linke dicht unterhalb ihres Abgangs eine derbe, gelbweiße, höckerige Einlagerung, die zu einer merklichen Stenosierung des Gefäßes geführt hat; das periphere Ende der Arterie ist frei von Veränderungen.

Daneben fanden sich ein hochgradiges Emphysem mit schwerer chronischer Bronchitis, eine cyanotische Muskatnußleber und cyanotische Induration der Milz, eine Pyelonephritis der rechten Niere; die linke Niere zeigte neben den schon makroskopisch deutlichen Merkmalen der Stauung mikroskopisch eine beginnende interstitielle Nephritis.

Epikritisch betrachtet stellen sich die in Frage stehenden Anfälle von Angina pectoris und angiospastischen Zuständen der Peripherie folgendermaßen dar: Patient litt an zwei verschiedenen Arten von Anfällen, die aber meist ineinander übergingen. 1. an völlig spontan auftretenden, heftigen, mit Todesangst verbundenen Schmerzen in der Magengrube, die, meist nachts rezidivierend, stets von totaler Asphyxie, Taubheit und Parästhesien der Finger und einzelnen Stellen des Gesichts (Nase, Wangen) begleitet waren; seltener traten dieselben subjektiven und objektiven Erscheinungen auch an den Füßen auf. Während dieses Anfalls steigt der (systolische) Blutdruck ${ }^{1}$ ) bedeutend (von $140 \mathrm{~mm}$ hg auf $165 \mathrm{~mm}$ ), die Pulsfrequenz nimmt ein wenig zu, die Atmung ist eher etwas verlangsamt. Die Temperatur stieg unter Frost beim ersten Anfall bis auf $37,2^{\circ}$. (Später auftretende höhere Temperaturen sind wohl ausschließlich auf Rechnung der gleichzeitig bestehenden Pyelitis zu setzen.) Hörte der Anfall nach 1-2 Stunden auf, so nahmen die vorher asphyktischen Hände eine hellrote Farbe und erhöhte Wärme an.

2. litt Patient an - nicht beobachteten, nur anamnestisch geschilderten - leichteren Anfällen: besonders bei Einwirkung von Kälte tritt eine mit Taubheit, Kältegefühl und Parästhesien verbundene Asphyxie der Finger ein, die von mäßigem Unbehagen und Sensationen auf der Brust und Magen begleitet ist. In letzter Zeit waren die letzteren Anfälle selten rein aufgetreten, sondern immer mehr in die erstere Form übergegangen.

Die Diagnose der Angina pectoris vera als der einen Komponente der Anfälle bedarf nach ihrer Art, dem Ausgang in plötzlichen Tod und der Konstatierung einer Sklerose der Coronararterien keiner weiteren Begründung.

Fall 2. Insufficientia cordis chronica. Sclerosisarter. coronar. Angina pectoris. Vasomotorisch-neurotische Erscheinungen der Peripherie (Hände, Gehirn und Gesicht.) Arthritis urica. Mors subita.

J. B., 47 Jahre alt, Schultheiß, leidet seit etwa vier Jahren an Herzbeschwerden, Atemnot, Herzklopfen, leichten Schwellungen der Füße. Seit Jahren kürzere Anfälle von Herzschmerz mit lebhaftem Angstgefühl, besonders nachts. In letzter Zeit haben die Anfälle folgenden Charakter angenommen: er empfindet das ihm wohlbekannte Gefühl der Herzangst und des Schmerzes über dem Herzen, dazu tritt leichter Kopfschmerz und Schwindel mit "Schwarzwerden vor den Augen", aber nie Bewußtlosigkeit. Im Anfall hämmernde Herztätigkeit. Oft treten zu diesem Anfall noch folgende Erscheinungen an den Fingern und Händen: die Finger "sterben $a b^{4}$, werden weiß, kalt und total gefühllos, die Nägel bläulich, wie die einer Leiche. Nachdem der Anfall (etwa in zehn Minuten) vorüber ist, werden die Finger sehr rot und warm. Bisweilen treten aber diese Anfälle vom Absterben der Finger auf äußere Veranlassungen (Kältereize beim Waschen) auf, sind dann höchstens von etwas Schwindel, vielleicht auch Schwarzwerden

1) Die - sehr wesentliche - Bestimmung auch des diastolischen Drucks konnte deshalb leider nicht erfolgen, weil bei der kurzen Behandlungsdauer nur ein Anfall beobachtet wurde, die späteren nachts zwischen 2 und 4 Uhr erfolgten. 
vor den Augen begleitet, verlaufen aber ohne wesentliche, dem Kranken peinliche Empfindungen von Seiten des Herzens. In letzter Zeit zunehmende Dekompensation, früher starkes Potatorium.

Status. Sehr großer, kräftiger Mann, typische, umschriebene Acrocyanose der Wangen, der Nase und Ohren, subikterische Gesichtsfarbe. Oedeme beider Unterschenkel mit Pigmentation und Ulcusnarben. Die Atmung ist etwas mühsam und beschleunigt, $\mathrm{Pa}-$ tient vermag aber noch ziemlich tief zu liegen. Auf der Linge I und RHU die Erscheinungen der Stauungsbronchitis. Herz: (Orthopercut.) rechts über fingerbreit vom rechten Sternalrand, links bis fast zur vorderen Mammillarlinie reichend. Stürmische Aktion des rechten Herzens, Spitzenstof verbreitert, hebend, bis in die vordere Mammillarlinie reichend. Ueber der Spitze lautes, systolisches Geräusch, zweiter Ton sehr leise, manchmal geräuschähnlich. Keine besondere Akzentuation der zweiten Töne der Basis.

Aktion sehr irregulär, zahlreiche Extrasystolen, kaum beschleunigt, am Radialpuls infolge Ausfallens vieler Extrasystolen scheinbar verlangsamt (Frequenz am Herzen 76, an der Radialis 56 p. M.), bisweilen reine Bigeminie auf kurze Pulsfolgen, bisweilen auffallend lange postextrasystolische Pausen.

Blutdruck (nach Riva-Rocci) systolisch: $145-150 \mathrm{~mm} \mathrm{hg}$, diastolisch : 75-78 $\mathrm{mm} \mathrm{hg}$; Pulsdruck also durchschnittlich $70 \mathrm{~mm} \mathrm{hg}$.

Venenpulse: Rechte Vena jugularis sehr stark erweitert und pulsierend, nach Sphygmogramm typisch zweigipfliger Puls, rein herzsystolisch; linke Vena jugularis nur wenig erweitert und pulsierend.

Abdomen relativ wenig aufgetrieben, geringer Meteorismus, kein Ascites; Leber 3-4 Finger breit den Rippenbogen überragend, hart, kein Leberpuls. Milz nicht vergrößert.

Am Nervensystem keine besonderen Veränderungen.

Urin etwas trüb, braunrot, Menge 600-1000, spez. Gewicht 1020, reichlich Eiweiß (2\% nach Esbach), kein Zucker. Im Sediment reichlich hyaline und granulierte Zylinder, Epithelien, keine Erythrocyten.

Ordination: 'Tct. Strophanti dreimal 10 Tropfen. (Patient hat bisher Digitalis genommen.)

16. Januar. Status idem. Immer etwas cyanotisch, Nachtruhe leidlich. Heute beim Waschen mit kaltem Wasser bekam Patient folgenden Anfall: Die Finger wurden eiskalt und taub, kriebelten; sie waren sehrblaß, die Nägel bläulich. Zugleich empfand Patient Schwindel, es wurde ihm schwarz vor den Augen, dabei leichtes Herzklopfen und leichter Schmerz über dem Herzen, weder subjektiv noch objektiv das Bild eines typischen Angina-pectorisanfalles. Patient geht ins Bett, wärmt sich die Hände, diese werden dann zuerst cyanotisch und darauf lebhaft hellrot und warm. Dabei ist die Herzaktion, was Frequenz anbetrifft, nicht alteriert, 54 in der Minute am Radialpuls (cf. das Ausfallen der Extrasystolen). Der Puls ist klein, das Rohr eng, rigide, der Druck deutlich vermehrt.

18. Januar. Allmähliche Kompensierung, Diurese steigt, subjektives Befinden ganz gut. Beim Waschen mit kaltem Wasser regelmäßig plötzlich Anfall von doigts morts, dazu Schwindel, Schwarzwerden vor den Augen, ganz leichtes anginöses Gefühl. Beim Waschen mit warmem Wasser fallen diese sonst stets morgens rezidivierenden Anfälle sofort weg.

24. Januar. Keine angioneurotischen Anfälle mehr.

27. Januar. Typischer Gichtanfall in der linken großen Zehe, in drei Tagen ablaufend.

3. Februar. Bisher keine Anfälle von Angina pectoris und vasomotorischen Störungen der Finger mehr. Mittags im Bett, nach ziemlich gutem Befinden vorher, fällt Patient plötzlich zurück, wird tief cyanotisch, röchelt einige Male und ist innerhalb weniger Sekunden tot.

Die Obduktion (Prof. v. Baumgarten) eroab bezüglich des Herzens folgendes: Das fast ganz horizontal liegende Herz, dessen vordere Fläche $\mathrm{zu}^{2 / 3}$ vom rechten, $\mathrm{zu} 1 / \mathrm{s}$ vom linken Ventrikel gebildet wird, ist bedeutend nach links (fast vordere Mammillarlinie) und nach rechts (zwei Finger breit vom rechten Sternalrand) verbreitert, seine Form ist mehr kugelig als konisch, Länge $15 \mathrm{~cm}$, Breite $12 \mathrm{~cm}$; leichte Schrumpfung der beiden Zipfel der Mitralkappe, besonders im Höhendurchmesser, geringe Stenose und Insuffizienz des Ostiums. Aortenklappen völlig normal, das Ostium valv. tricuspidal. abnorm weit. Der linke Ventrikel ist hypertrophisch (Wanddicke ohne Trabecul. carneae $12 \mathrm{~cm}$, nur wenig dilatiert). Der linke Vorhof stark dilatiert und hypertrophisch. Der rechte Ventrikel ist enorm dilatiert und auch deutlich hypertrophiert, ebenso Dilatation und Hypertrophie des rechten Vorhofs.

Das Herzfleisch zeigt auf Flachschnitten makroskopisch keine besonderen Veränderungen. Die Coronarterien sind beide für eine Stahlsonde gut permeabel; nach dem Aufschneiden zeigt namentlich die linke A. coronaria zahlreiche gelbliche, sklerotische Verdickungen. Eine derselben, die umfangreichste, hat etwa in der
Mitte des Ramus descendens der linken A. coronar. eine erheblichere Verengerung des Gefäßlumens bewirkt, die aber dem Einführen der Stahlsonde den Durchgang nicht verwehrte. Im übrigen fanden sich braune Induration der Lungen, stark venöse Hyperämie der Nieren, Uratniederschläge in beiden Nieren, cyanotische Induration der Milz, venöse Hyperämie der Leber mit Perihepatitis; im Gelenk der linken großen Zehe die Residuen des frischen Gichtanfalles.

Fall 2 ist, epikritisch betrachtet, dem ersten Fall außerordentlich ähnlich, nur tritt die Trennung von typischer Angina pectoris des Herzens und angiospastischen Zuständen der Peripherie noch schärfer hervor: in den ersten Jahren bestehen reine, mäßjig schwere Anfälle von Angina pectoris ohne sonstige vasomotorische Erscheinungen. Später treten zu diesen Herzerscheinungen Gefäßkrämpfe der Peripherie, die sich in den Erscheinungen der Hirnanämie (Ohnmachtsanwandlungen, Schwarzwerden vor den Augen), gleichzeitiger hochgradiger Asphyxie der Hände (weniger der Füße) und nachfolgender Hyperämie derselben ausdrückten. Dieser Komplex der peripheren vasomotorischen Störung allein tritt nun während der klinischen Behandlung durchaus in den Vordergrund; er wird - wie harmlose genuine Zustände dieser Art - stets ausgelöst durch das Waschen mit kaltem Wasser etc. und ist nur von leichtem Beklemmungsgefühl und Schwindel begleitet. Während der gemischten Anfälle veränderte sich die Schlagfolge des Pulses nicht wesentlich, der (im Anfall nicht gemessene) Druck war augenscheinlich vermehrt, subjektiv bestanden Herzpalpitationen, die Atmung war nicht beschleunigt.

Auch in diesem Fall kann an der Diagnose der Angina pectoris nach der Art der Anfälle, des plötzlichen Todes und dem Obduktionsbefund (stenosierende Coronarsklerose) kein Zweifel sein.

Die geschilderten angiospastischen Zustände der Peripherie zeigten in beiden Fällen unbestreitbar die weitgehendste Uebereinstimmung mit dem von Nothnagel als Angina pectoris vasomotoria geschilderten Krankheitsbild. Bei beiden $\mathrm{Pa}$ tienten - bei der ersten sehr selten, beim zweiten häufig, fast regelmäßig - verliefen die leichteren, durch Kälte ausgelösten Anfälle genau so, wie sie Nothnagel in vier anscheinend herzgesunden Fällen beobachtet hat: mit anfallsweise auftretender Asphyxie der Hände, seltener der Füße und leichtem Gefühl von Beängstigung und Herzpalpitationen. Sie unterscheiden sich aber dadurch von den Nothnagelschen Fällen, daß sie beide mehr oder weniger fließende Uebergänge und Kombinationen von echter Angina pectoris mit peripheren $\mathrm{Ge}$ fäßkrämpfen darstellen und klinisch sowie anatomisch das Signum der tödlichen Angina pectoris infolge von Coronarsklerose trugen.

Nothnagel hat den von ihm gefundenen Symptomenkomplex der Angina pectoris vasomotoria in allen seinen Fällen für ein gutartiges, rein nervöses Leiden der peripheren Gefäbe erklärt, das erst sekundär - auf noch zu erörternde Weise - subjektive Herzerscheinungen auslöse. Einige seiner Fälle zwingen aber den Leser dazu, an der Annahme, daf es sich wirklich um gesunde Herzen, nicht um echte Angina pectoris gehandelt habe, ein wenig zu zweifeln; vor allem der Fall 3: bei einer 63 jährigen Frau kommt es zusammen mit peripheren Gefäßkrämpfen zu „fürchterlichem Herzklopfen“, „lebhafter Beängstigung, heftigem Druckgefühl, als ob Steine auf der Brust lägen". Fall 4, 46jähriger Sackträger hat während der Vertaubung der Finger "ein unsägliches Gefühl von Beängstigung, ihm war, als müsse er vergehen, sterben“. Die Beobachtungsdauer aller vier Fälle war außerdem eine relativ kurze, jedenfalls zu kurz, als daß der die Diagnose der Angina pectoris besiegelnde Exitus subitus schon hätte erwartet werden können.

Die Angabe, daß das Herz bei allen Patienten in den anfallsfreien Intervallen völlig normale Verhältnisse gezeigt habe, ist für uns - abgesehen von dem Fehlen genauerer Angaben über die Herzbefunde - trotz der Autorität des Beobachters vielleicht nicht unbedingt bindend, wenn wir bedenken, daß im Jahre 1867 die Kenntnis und die klinische Diagnose der arteriosklerotischen Herzveränderungen in den anfallsfreien Zeiten weit unsicherer war, als heute. Auch die syphilitische Aetiologie, dies für die Diagnose der echten Angina pectoris 
im mittleren Alter so überaus wichtige Moment, war noch nicht bekannt und blieb demzufolge unberücksichtigt.

Jedenfalls. beweisen meine Fälle, dab das ganze Symptomenbild der Angina pectoris vasomotoria auch auf dem Boden oder besser als Begleiterscheinung einer echten coronarsklerotischen Angina pectoris auftreten kann, und daß es nicht immer, wie Noth. nagel annahm, auf dem Boden einer Neurasthenie oder verwandter Zustände erwachsend eine harmlose Neurose der peripheren GefäBe darstellt, die erst sekundär zu leichter Alteration des Herzens führt.

Es fehlten übrigens - das sei ausdrücklich hervorgehoben bei beiden Patienten irgendwelche Symptome einer Neurasthenie oder Hysterie absolut, ein Beweis mehr dafür, daß es sich bei ihnen nicht um eine zufällige, an sich nicht zusammenhängende Kombination von Angina pectoris und einer einfachen vasomotorischen Neurose handelte. Auch fehlten bei beiden Patienten gewisse, als disponierend für Angioneurosen angesehene Berufschädlichkeiten. Schließlich fehlte auch ein disponierendes Moment, auf das man bisher noch nicht geachtet hat, das mir aber für die genuinen vasomotorischen Neurosen nicht unwesentlich erscheint, die spezielle hereditäre Belastung. ${ }^{1}$ )

Die Frage: In welcher Beziehung stehen die Anfälle von Angina pectoris oder die anginösen Empfindungen (Nothnagel) zu den Angiospasmen der Peripherie? hat schon den ersten Beobachter der Angina pectoris vasomotoria sehr interessiert, scheint aber zurzeit eine befriedigende Beantwortung noch nicht zuzulassen. Unzweifelhaft bestand in unseren Fällen ein innerer Zusammenhang zwischen den beiden Gefäßstörungen: die leichten Fälle von Beängstigung konnten als Folgen des peripheren Gefäßkrampfes durch Kälteapplikation auf die Peripherie ausgelöst werden; in den schweren Anfällen schienen allerdings die zentralen (cardialen) und peripheren Krämpfe gleichzeitig und völlig koordiniert aufzutreten. Für die erstere Form läge es nun nahe, aus der starken Steigerung des Blutdruckes zu vermuten, daß etwa der plötzlich auftretende Widerstand in Gestalt einer ausgedehnten Gefäß. verengerung der Peripherie für das Herz einen lebhaften Shock, eine stark, unvermittelt zu leistende Mehrarbeit bedeuten. Man könnte dann annehmen, daß, wie es bei sklerotisch veränderten und darum erfahrungsgemäß zum Spasmus neigenden Gefäßen der Peripherie bei vermehrter Arbeit der betreffenden Extremität zum schmerzhaften Gefäßkrampf kommen kann (cf. die Dysbasia angiosclerotica Charcot-Erb), auch die durch Sklerose disponierten Coronargefäße diese Mehrarbeit mit einem Krampf, d. i. der Angina pectoris beantworteten. Aber man sehe sich bei dieser Deutung vor und sei sich klar, daß sie durchaus hypothetisch ist! Es handelte sich bei unseren Fällen, soweit sich nachweisen lieb, nur um einen gesteigerten Maximaldruck. Dieser beweist und involviert aber durchaus noch nicht die angenommene Mehrarbeit für das Herz, die Conditio sine qua non für die genannte Hypothese. Auf diese Mehrarbeit können wir nur schließen, wenn auch der diastolische Druck entsprechend erhöht gefunden wird. Daß dies letztere nun bei schwerer Angina pectoris mit gewaltiger systolischer Hochspannung (bis $175 \mathrm{~mm} \mathrm{hg)} \mathrm{absolut} \mathrm{nicht} \mathrm{der} \mathrm{Fall} \mathrm{zu} \mathrm{sein}$ braucht, daß der diastolische Druck im Anfall niedrig sein kann, lehrte mich ein neulich beobachteter Fall von Angina pectoris bei Aorteninsuffizienz. Wir sehen daraus, daß man die oben erörterte Hypothese des Zusammenhanges zwischen Angina pectoris vera und peripherem Gefäßkrampf noch mit grofer Vorsicht und Reserve betrachten muß. Jedenfalls mahnen uns die beiden Fälle bei anscheinend primären, „nervösen“, vasomotorischen Krisen der Peripherie der typischen Angina pectoris vasomotoria immer die Möglichkeit, der echten Angina pectoris als eine - und zwar die klinisch wesentlichste Komponente ins Auge zu fassen.

1) Vor kurzem beobachtete ich z. B. eine Familie, in der die Großmutter (mütterlicherseits), die Mutter und drei Töchter im Alter von 10-20 Jahren seit früher Jugend an angiospastischen Akropärästhesien, Asphyxie der Finger etc. litten. Im übrigen bestanden bei allen hysteroneurasthenische Symptome. Daß auch für die Raynaudsche Krankheit die familiäre Disposition sehr in Frage kommt, lehrt eine interessante (noch nicht veröffentlichte) Beobachtung von $\mathbf{P}$. Linser.
Das gilt ganz besonders für die Fälle von „intermittierendem Hinken", die nicht die unteren Extremitäten, sondern den Darm, die oberen Extremitäten, das Gesicht und die Augen betreffen. Für die Claudication intermittente der Beine steht ja die häufige Kombination mit echter Angina pectoris durch die grundlegenden Beobachtungen Erbs fest.

Unter drei an der Medizinischen Klinik in letzter Zeit beobachteten Fällen von Claudicatio intermittens der Arme, des Gesichtes und der Augen, zeigte einer in charakteristischer Weise den peripheren Angiospasmus stets im Angina pectorisAnfall. Die kurze Schilderung des Falles möge hier folgen:

Fall 3. Aneurysma der aufsteigenden Aorta, Angina pectoris mit Anfällen von rechtseitiger Amaurose.

Christine W., 43 Jahre, die einer luetischen Infektion dringend verdächtig ist (die ersten vier Geburten Aborte, dann drei sofort post partum sterbende Kinder, dann drei schwächliche, lebende Kinder) klagt seit etwa zwei Jahren über Atemnot, Schwindel, Herzklopfen und Kräfteverfall; besonders beängstigt ist sie durch folgende Anfälle: sie empfindet plotzlich ein heißes, brennendes Gefühl, das von der Herzgegend nach unten und oben zieht, mit heftiger Angst und Vernichtungsgefühl verbunden ist, dabei starkes Herzklopfen. Dabei bemerkt sie, daß ihr vor dem rechten Auge "schwarz wird“, daß sie während einiger Minuten auf dem rechten Auge total erblindet ist. Diese Anfälle wiederholen sich alle Wochen und öfter und werden von der Patientin aufs äußerste gef ürchtet.

Status: 4. Mai: Die etwas abgemagerte, anämische Patientin zeigt eine mäßige Verbreiterung des Herzens nach links und rechts, über und neben dem Sternum eine deutliche Dämpfung, über der starkes systolisches und diastolisches Schwirren palpabel ist. An allen Aortenauskultationspunkten, nach oben zunehmend, ein lautes systolisches und noch lauteres diastolisches Geräusch. Im Rontgenbild sieht man über dem Schatten des rechten Vorhofes eine nach rechts konvexe, bucklige, stark pulsierende Vorwolbung, die besonders im zweiten schrägen Durchmesser sichtbar wird; über derselben Gefäßschatten wieder von normalem, ziemlich geringem Umfange.

Die rechte Carotis zeigt geringere Pulsation als die linke; ebenso die rechte $A$. radialis < als die linke. Blutdruck $160 \mathrm{~mm} \mathrm{Hg}$, Aktion regulär, aequal, Abdomen ohne Besonderheiten, keine Leberschwelluug. Nervensystem: keine organischen, speziell keine tabischen Erscheinungen; keine neurasthenischen Züge. Larynx: in den ersten Tagen Entwicklung einer rechtseitigen Recurrensparese.

Augenhintergrund (Priv.-Doz. Dr. Fleischer): Papillen normal, Arterien und Venen von gleicher Beschaffenheit und Füllung.

11. Mai: Spät abends im Bett plötzlicher Anfall: Heftiger, nach allen Seiten ausstrahlender Schmerz über dem Her zen, Todesangst, zugleich vollständiges "Blindwerden“ auf dem rechten Auge; auch die mäßige Heiserkeit soll im Anfall zu einer totalen geworden sein; dabei nach An gabe der Pflegerin blasses, sehr ängstliches Aussehen und gut fühlbarer, regelmäßiger, rechtseitiger Radialpuls. Dauer des Anfalles etwa eine Viertelstunde. Keine ausstrahlenden Schmerzen, keine Asphyxie, keine Parese des rechten Armes und Hand im Anfall. Am nächsten Morgen rechts und links gleiches und normales Sehvermögen; der Augenspiegelbefund zeigt völlig gleiche Gefäßverhältnisse auf beiden Seiten; auch die Heiserkeit ist fast verschwunden und laryngoskopisch die rechtseitige Rekurrensparese weit geringer als vorher.

12. Mai: Patientin verließ, durch den Anfall lebhaft beunruhigt, die Klinik.

Die Diagnose des Aneurysmas der aufsteigenden Aorta war durch den Befund der Perkussion und Auskultation, durch die Pulsdifferenz von Radialis und Carotis, sowie durch das Röntgenbild, schließlich auch durch die sich rasch entwickelnde Rekurrensparese gesichert. Ebenso kann an der Auffassung der beschriebenen Anfälle als Angina pectoris vera kein Zweifel bestehen. Eigentümlich an diesem Fall ist die Kombination des Angina pectoris-Anfalles mit einer vorübergehenden Amaurose des rechten Auges. Während in Fall 2 der stenocardische Anfall von einem allgemeinen Gefäskrampf des Gehirnes und vorübergehender Amblyopie auf beiden Augen gefolgt war, müssen wir hier als Ursache der paroxysmalen einseitigen Erblindung einen vorübergehenden Gefäßverschluß, höchstwahrscheinlich spastischer Natur, in der Ketinalarterie annehmen, deren zuführende Hauptarterie, die A. carotis, dauernd ein geringeres Volumen zeigte, als die der anderen Seite; daß es sich nicht um eine dauernde Schädigung der retinalen Durchblutung 
handelte (Thrombose, Embolie), zeigte der völlig normale Visus und opthalmoskopische Gefäßbefund bald nach dem Anfall.

Es handelte sich also, auch wenn die Untersuchung des Augenhintergrundes im Anfall nicht erfolgen und diese Annahme stützen konnte, höchstwahrscheinlich um ein analoges Verhalten, wie in dem bekannten Fall von Wagemann; nur mit dem Unterschied, daß in jenem Falle der vorübergehende Spasmus der A. retinae nicht die Begleiterscheinung eines Angina pectoris-Anfalles war, sondern, wie das intermittierende Hinken, ausschlieblich lokale, periphere Ausfallserscheinungen bereitete. Genau dasselbe Verhalten, vorübergehendes Vertauben und Erlahmen der rechten Hand, der rechten Gesichtshälfte und vorübergehende Amblyopie des rechten Auges, fanden wir auch bei einem Patienten unserer Klinik, ohne daf es auch in diesem Falle gleichzeitig zu einem stenocardischen Anfalle kam. Durch die Kombination des Angina pectorisAnfalles mit den Zeichen des spastischen Gefäßverschlusses einer Gehirnarterie tritt unser Fall in direkte Analogie zu den beiden ersten. Er ähnelt auch dem von v. Schroetter beschriebenen, in dem es bei dem gleichen Grundleiden - Aneurysma - im Angina pectoris-Anfall zu einer totalen Asphyxie der Extremität (Hand) kam, deren Arterienpuls dauernd ein geringeres Volumen zeigte, als der der anderen Seite. Wahrscheinlich gehört in diese Kategorie auch der interessante Fall von $H$. Herz (halbseitige Asphyxie im stenocardischen Anfall), wenn auch Verfasser über den Zustand (insbesondere über eine Differenz) der Arterienpulse beider Körperhälften nichts berichtet.

Nachtrag bei der Korrektur: Nach Abschluß dieser Arbeit hatte ich durch Zufall Gelegenheit, noch zwei Fälle zu beobachten, von denen der eine als $r$ in nervöse Angina pectoris vaso. motoria im Sinne Nothnagels, der andere aber - wie die drei beschriebenen Fälle - als Kombination dieses Zustandes mit echter Angina pectoris aufzufassen ist. Im ersteren Falle handelt es sich um eine 26jährige Frau, die ohne erkennbare Ursache seit zwei Jahren an "Absterben der Hände und Füße“ leidet. Diese Anfälle wiederholen sich fast jeden Abend zwischen $81 / 2$ und 9 Uhr und sind von Präcordialangst begleitet, "es ist ihr, als schnüre es ihr das Herz ab". Dauer des Anfalles eine halbe bis eine Stunde. In der Klinik verschwand diese Art der Anfälle sofort und wurde substituiert durch eine andere Art, durch ein lautes Luftschlucken, Singultus und Ructus typisch-hysterischer Art, die pünktlich zu der gewohnten Zeit der vasomotorischen Anfälle, um $81 / 2$ Uhr abends, auftreten und durch Valeriana sofort zu beseitigen sind. Herz, Gefäße (speziell Blutdruck), Abdominalorgane ohne Veränderung; keine hysterischen Stigmata, aber ausgesprochen hysterische Psyche.

Der zweite Fall, eine 56 jährige Frau, leidet seit einigen Monaten, besonders nachts, an anfallsweise auftretender Asphyxie zuerst der Beine („bis zum Knie kalt und pelzig“), dann der Hände, dabei Schwindel, Schwarzwerden vor den Augen, manchmal heftiges Erröten und Heißwerden des Gesichtes. Während der Anfälle stets starker Druck auf dem Herzen, Brennen, Vernichtungsgefühl. Objektiv fanden sich bei der Patientin: Permanent auffallend kühle, blasse Exxtremitäten, desgleichen das Gesicht sehr kühl. Hyper. trophie und Dilatation besonders des linken Herzens; Klappen des zweiten A ortentons, enge, rigide und geschlängelte Arterien; Blutdruck: systolisch $148 \mathrm{~mm}$, diastolisch $92 \mathrm{~mm} \mathrm{hg} \mathrm{Riva-Rocci,} \mathrm{also}$ erhöhter systolischer Blutdruck und vermehrter Pulsdruck. Die Fußpulse der A. tibialis postica fehlen. Urin frei von Eiweiß, im Sediment aber einige Zylinder. Dazu chronische Bronchitis, Stauungsleber. Keine Anzeichen einer Neurose; Symptome des intermittierenden Hinkens fehlen.

Ich glaube, daß man den letzteren Fall wegen der charakteristischen Veränderungen des Herzens und der Gefäße den drei oben beschriebenen direkt anreihen und bei ihm ebenfalls die Diagnose Angina pectoris vera kombiniert mit vasomotorischen Krampfzuständen der Peripherie stellen darf.

Literatur: 1. H. Nothnagel, Angina pectoris vasomotoria. Deutsches Archiv für klinische Medizin Bd. 3. - 2. Derselbe, Zur Lehre von den vasomotorischen Neurosen. Ibidem Bd. 2. - 3. E. B amberger, Kasuistische Beiträge zur Symptomatologie der Herzneurosen. Wiener klinische Wochenschrift 1888 , No. 31. - 4. v. Schrotter, Erkrankung der Gefäße. Nothnagels Handbuch - 5. Huchard, Traité clinique des mantis maladies du Cour P. Pasomotorischen Neurosen. - 7. J. Pal, Gefäßkrisen. Leipzig 1905. - 8. v. Frankl-Hochwart, Nothnagels Handbuch. - 9. Oppenheim, Lehrbuch der Nervenkrankheiten 1904 . 10. H. Herz, Zur Lehre von den Neurosen des peripheren Kreislaufapparates. BerlinWien 1902. 